\title{
Modeling of Temperature Effect on Modal Frequency of Concrete Beam Based on Field Monitoring Data
}

\author{
Wenchen Shan $\left(\mathbb{D},{ }^{1}\right.$ Xianqiang Wang, ${ }^{2}$ and Yubo Jiao ${ }^{1}{ }^{1}$ \\ ${ }^{1}$ College of Transportation, Jilin University, Changchun, Jilin 130025, China \\ ${ }^{2}$ Jiangsu Transportation Institute, Nanjing 211112, China \\ Correspondence should be addressed to Yubo Jiao; jiaoyb@jlu.edu.cn
}

Received 14 November 2017; Accepted 14 January 2018; Published 11 February 2018

Academic Editor: Giosuè Boscato

Copyright (C) 2018 Wenchen Shan et al. This is an open access article distributed under the Creative Commons Attribution License, which permits unrestricted use, distribution, and reproduction in any medium, provided the original work is properly cited.

\begin{abstract}
Temperature variation has been widely demonstrated to produce significant effect on modal frequencies that even exceed the effect of actual damage. In order to eliminate the temperature effect on modal frequency, an effective method is to construct quantitative models which accurately predict the modal frequency corresponding to temperature variation. In this paper, principal component analysis (PCA) is conducted on the temperatures taken from all embedded thermocouples for extracting input parameters of regression models. Three regression-based numerical models using multiple linear regression (MLR), back-propagation neural network (BPNN), and support vector regression (SVR) techniques are constructed to capture the relationships between modal frequencies and temperature distributions from measurements of a concrete beam during a period of forty days of monitoring. A comparison with respect to the performance of various optimally configured regression models has been performed on measurement data. Results indicate that the SVR exhibits a better reproduction and prediction capability than BPNN and MLR models for predicting the modal frequencies with respect to nonuniformly distributed temperatures. It is succeeded that temperature effects on modal frequencies can be effectively eliminated based on the optimally formulated SVR model.
\end{abstract}

\section{Introduction}

With the development of advanced techniques in sensing, data acquisition, computing, and information management, structural health monitoring (SHM) systems have been widely implemented to diagnose structural condition [1-3]. Vibration-based damage identification, as an important part of SHM system, has been most widely investigated because of their capability for accurately identifying structural damage [4]. The basic concept underlying the use of vibration-based damage identification is that vibration properties (natural frequencies, mode shapes, and damping ratio) are functions of the physical properties (mass, stiffness, and damping) of structure $[5,6]$. The measured changes in dynamic parameters can be used to evaluate corresponding changes in physical properties that indicate the structural damage. However, dynamic parameters are inevitably affected by environmental and operational conditions (temperature, humidity, wind, traffic load, etc.). Changes of dynamic parameters caused by environment may lead to a false damage identification result [7-10].

Modal frequency is the most widely used dynamic parameter to identify structural damage because it is easy to be measured with high precision [11]. Extensive researches have demonstrated that temperature change is the most important source that causes the variation in modal frequencies of structures [12]. Researchers from Los Alamos National Laboratory find that the first three frequencies of Alamosa Canyon Bridge vary about $4.7 \%, 6.6 \%$, and 5.0\% during 24 hours, in which the temperature of the bridge deck changes by approximately $22^{\circ} \mathrm{C}[13,14]$. Peeters et al. $[15,16]$ report that the first four modal frequencies of Z24 Bridge vary by $14 \%-18 \%$ during 10 -month monitoring period, and this variation is more significant than the change of $10 \%$ caused by destructive damage. Desjardins et al. [17] make a continuous monitoring for the modal frequencies and average girder temperature of Confederation Bridge. The modal frequencies are reduced by $4 \%$ when the temperature 
varies from $-20^{\circ} \mathrm{C}$ to $25^{\circ} \mathrm{C}$. Askegaard and Mossing [18] continuously monitor a three-span reinforced concrete (RC) bridge for three years, and the seasonal changes of modal frequencies reach as much as $10 \%$. Yuen and Kuok [19] extract the modal frequencies of a 22-storey RC building for one year using Bayesian spectral density approach. They find that the first three modal frequencies increase with an increase in ambient temperature. Chen et al. [20] explore the correlation between modal frequencies of Guangzhou New TV Tower and air temperature through more than $100 \mathrm{~h}$ measurement. Results show that modal frequencies are linearly dependent on air temperature. Saisi et al. [21, 22] present the results of continuous dynamic monitoring for Gabbia Tower in Italy during a period of 8 months. Identified natural frequencies are observed to vary by $5-11 \%$ when the measured temperatures range from $2^{\circ} \mathrm{C}$ to $45^{\circ} \mathrm{C}$. Ubertini et al. [23] monitor the modal frequencies of San Pietro Bell Tower during more than nine-month period. Temperature variation produces significant changes in natural frequencies, up to $16 \mathrm{MHz} /{ }^{\circ} \mathrm{C}$, while effects of air humidity were relatively marginal. As indicated by the prior researches, temperature significantly affects the modal frequencies. In addition, no such temperature dependence has been observed for the mode shapes and damping ratio, and hence the temperature effect on them can be generally ignored [12, 24]. For the reliable performance of vibration-based damage identification, it is of paramount importance to eliminate and discriminate the variations in modal frequencies due to temperature change from those caused by structural damage.

In order to eliminate the temperature effect on modal frequency, quantitative models between them are required to normalize the identified modal frequencies to an identical reference temperature [25-27]. Xia et al. [24] propose a simple linear regression model to correlate the air temperatures and modal frequencies of a RC slab based on laboratory monitoring data. Peeters and De Roeck [15] derive an autoregressive and moving average (ARMA) model to formulate the relationship between air temperature and modal frequencies for the Z24 Bridge. Moser and Moaveni [28] utilize several models (a static linear model, an ARX model, a bilinear model, and polynomials with various orders) to represent the relationship between the modal frequencies and measured temperatures. Ding and Li [29] propose a polynomial regression model to describe the frequency-temperature seasonal correlations of the Runyang Suspension Bridge. Ni et al. $[30,31]$ apply the support vector machine (SVM) and backpropagation neural network (BPNN) techniques to formulate regression models that quantified the temperature effect on modal frequencies of the cable-stayed Ting Kau Bridge. These studies mentioned above have proposed methods for predicting the modal frequencies of bridges, but none has compared the prediction accuracy of multiple linear regression (MLR), BPNN, and SVM methods. In addition, these regression models mainly focus on the relationships between modal frequencies and air temperature or structural temperatures measured at some surface points. They ignore the nonuniformly distributed temperatures in the cross-section of structure, which may lead to information loss in modeling the temperature effect on modal frequencies $[32,33]$.
In this paper, three regression models of predicting modal frequencies corresponding to nonuniformly distributed temperatures are built on measurements from a concrete beam during 40-day monitoring period. Prediction capabilities are compared in order to select the optimal model for eliminating temperature effect on modal frequency. The rest of the paper is organized as follows. The various regression algorithms that are adopted to predict the modal frequencies are first outlined. A concrete beam is constructed and the details are described. Principal component analysis (PCA) is performed to extract principal components from the measured temperatures. And the quantitative models using MLR, BPNN, and SVR are constructed by use of extracted principal components (PCs) of temperatures. Prediction capabilities of constructed regression models are studied and examined on training and test samples. Selected optimal model is later used to remove the variability of identified modal frequencies due to temperature effect. Lastly, results are summarized with important conclusions.

\section{Theoretical Background}

Modal frequency is directly related to the temperature distribution across the structure. This research proposes to employ internal distributed temperature measurements to predict the modal frequency of a concrete beam. The methodology is outlined in the form of a flowchart in Figure 1. Temperature measurements collected from monitoring are first preprocessed by PCA for dimensionality reduction. The PCs of temperatures are then supplied as input to statistical regression techniques to compute regression models. Prediction capabilities of regression models are examined and compared based on statistical indicators. The regression model with the best prediction accuracy is then used to predict modal frequency from collected temperatures, which have been preprocessed by PCA. Temperature effect on modal frequency is successfully eliminated using the regression model with the best prediction accuracy.

2.1. Principal Component Analysis (PCA). PCA is multivariate statistical tool that takes advantage of inherent correlations between variables for dimensionality reduction and feature extraction. It is a linear transformation mapping an original set of variables into a substantially smaller set of uncorrelated variations that represents most of information in original set of variables $[3,34]$. Using PCA, original set of correlated variables $X \in R^{n}$ in an $n$-dimensional space can be transformed into a new set of uncorrelated variables $Y \in R^{m}$ in an $m$-dimensional $(m<n)$ orthogonal space by the application of the following equation:

$$
Y=\Gamma^{T} X
$$

where $\Gamma(n \times m)$ is a transformation matrix that applies an orthogonal rotation to the original coordinate system. Through the singular value decomposition for the covariance matrix of original variables $X$, we can obtain

$$
X X^{T}=U \Lambda U^{T},
$$




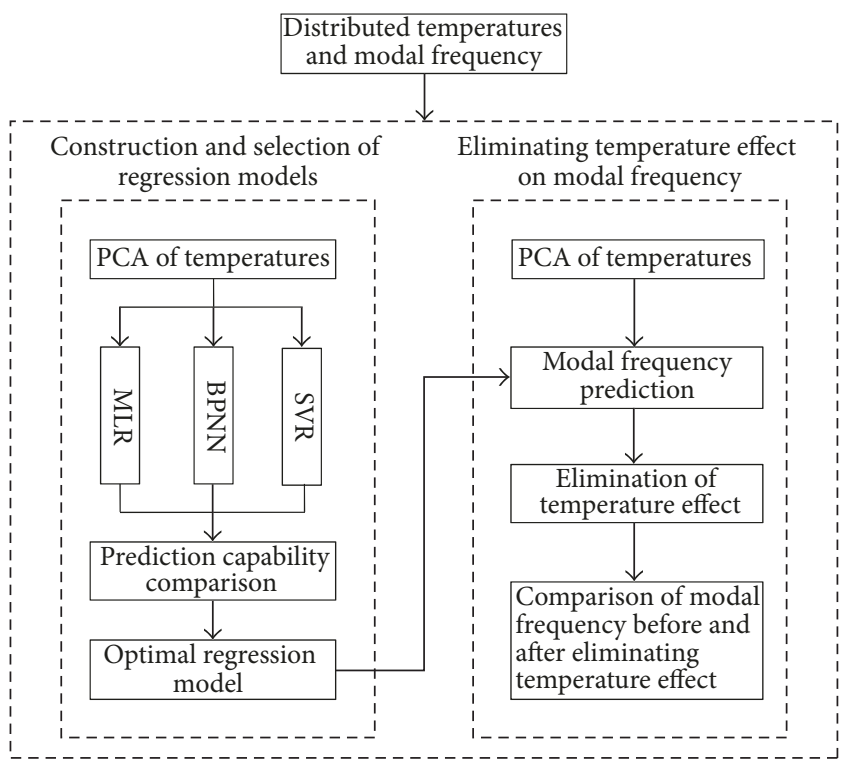

FIGURE 1: Technical flowchart of proposed method in this paper.

where $U$ is the orthogonal eigenvector matrix with $U^{T} U=I$ and $\Lambda$ is a diagonal matrix composed of singular values as follows:

$$
\Lambda=\left[\begin{array}{cccc}
\lambda_{1} & 0 & \cdots & 0 \\
0 & \lambda_{2} & \vdots & 0 \\
\vdots & \vdots & \ddots & \vdots \\
0 & 0 & \cdots & \lambda_{n}
\end{array}\right] \text {, }
$$

where the singular values rank in descending order $\lambda_{1} \geq$ $\lambda_{2} \geq \cdots \geq \lambda_{n} \geq 0$. They represent the variances of principal components, and the small singular values are not relevant to explain the overall variance of data set. The proportion of original variables explained by the first $m$ principal components is defined as

$$
\beta_{m}=\frac{\sum_{j=1}^{m} \lambda_{j}}{\sum_{i=1}^{n} \lambda_{i}}
$$

where $\beta_{m}$ is accumulated variance contribution rate and decides the number of selected principal components. Generally, if $\beta_{m} \times 100 \% \geq 85 \%$, the transformation matrix $\Gamma$ could be obtained by the first $m$ column vectors in orthogonal eigenvector matrix $U$. Once $m$ has been chosen and orthogonal transformation matrix $\Gamma$ has been determined, variables in principal space can be calculated by (1).

2.2. Multiple Linear Regression (MLR). MLR is an extension of simple linear regression for the purpose of predicting dependent variables by multiple explanatory variables [35]. When a dependent variable $y$ is linearly related to $n$ explanatory variables, the general form of MLR model can be expressed as

$$
y=\beta_{0}+\beta_{1} x_{1}+\beta_{2} x_{2}+\cdots+\beta_{n} x_{n}+e,
$$

where $y$ is the predicted value of dependent variable, $\beta_{0}$ is the intercept and $\left(\beta_{1}, \beta_{2}, \ldots, \beta_{n}\right)$ are regression coefficients associated with the explanatory variables $\left(x_{1}, x_{2}, \ldots, x_{n}\right)$, and $e$ is random error with mean zero and variation $\sigma^{2}$. Based on the data from $n$ measurements, unknown regression coefficients can be determined using least-squares method. In this study, $y$ represents the modal frequency at specific time, and $\left(x_{1}, x_{2}, \ldots, x_{n}\right)$ represent PCs extracted from measured temperatures in cross-section at mid-span of concrete beam.

2.3. Back-Propagation Neural Network (BPNN). Artificial neural network (ANN) is a functional abstraction from biologic neural structure, which can process complex nonlinear relationships among several variables through learning [36]. Therefore, it provides a powerful tool for modeling the relationship between modal frequencies and distributed temperatures. As one of the widely used ANN structures, BPNN is established through forward transfer of information and back-propagation of training error. The biases and weights are constantly adjusted to minimize target error through gradient descent algorithm. An evaluator, the sum of square error (SSE) between actual and target outputs, is taken as the objective function of BPNN model, as shown in

$$
\operatorname{SSE}=\sum_{k=1}^{p} \sum_{m=1}^{n}\left(O_{k m}-T_{k m}\right)^{2}
$$

where $O_{k m}$ and $T_{k m}$ are the actual and target output of $m$ th node in output layer for $k$ th pattern, respectively, $n$ is the number of outputs, and $p$ is the number of patterns.

The typical two-layer BPNN contains an input layer, hidden layer, and output layer. The transfer function for hidden layer is taken as a tan-sigmoid function and that for the output layer is a linear function. In this research, BPNN is simulated using MATLAB's neural network toolbox, and the "traingdx" and "learngd" functions are chosen as training function and learning function, respectively [37]. An important parameter in BPNN is the optimal number of nodes in the hidden layer. It is optimally determined through trials and validation errors. In order to avoid the underfitting and overfitting phenomena, an early stopping technique is employed. Training process is stopped when the errors on validation data increases for a specific number of iterations. The parameters (weights and biases) of BPNN model are determined as those associated with the minimum of validation error.

2.4. Support Vector Regression (SVR). Support vector machine (SVM) is a newly emerging learning technique following the structural risk minimization (SRM) principle rather than the common empirical risk minimization (ERM) principle. It transforms sample data to a higher dimensional feature space and defines the optimal linear hyperplane to minimize the upper bound on the generalization error [3, $30,38]$. SVR refers to the regression model of SVM. It is to transform the nonlinear relationship in original space into linear relationship in a feature space so as to discover relationship more easily. 
Consider a set of training data $S=\left\{\left(\mathbf{x}_{1}, y_{1}\right),\left(\mathbf{x}_{2}, y_{2}\right)\right.$, $\left.\ldots,\left(\mathbf{x}_{n}, y_{n}\right)\right\} \in R^{P} \times R$, where $\mathbf{x}_{i}$ is a $P$-dimensional input vector and $y_{i}$ is the corresponding scalar output in original space. For linear regression problem in the feature space, the linear estimation function is described as

$$
f(x)=\langle\mathbf{w}, \varphi(\mathbf{x})\rangle+b,
$$

where $\mathbf{w}$ and $b$ are weights and thresholds, respectively, $\varphi$ is the mapping function transforming input vector to feature space, and $\langle *, *\rangle$ denotes the inner product.

The SRM principle is adopted in SVR to avoid overfitting and improve generalization performance. The optimization object is set as

$$
\begin{array}{cl}
\text { Minimize } & \frac{1}{2}\|\mathbf{w}\|^{2}+C \sum_{i=1}^{n}\left(\xi_{i}+\xi_{i}^{*}\right) \\
\text { Subject to } & y_{i}-\left\langle\mathbf{w}, \varphi\left(\mathbf{x}_{i}\right)\right\rangle-b \leq \varepsilon+\xi_{i} \\
& \left\langle\mathbf{w}, \varphi\left(\mathbf{x}_{i}\right)\right\rangle+b-y_{i} \leq \varepsilon+\xi_{i}^{*} \\
& \xi_{i}, \xi_{i}^{*} \geq 0,
\end{array}
$$

where $\varepsilon$ is loss function, $C$ is penalty coefficient, and $\xi_{i}, \xi_{i}^{*}$ are slack variables.

Solution of (8) under constraints of (9) is achieved by introducing the Lagrange multipliers and using the duality principle.

$$
\begin{aligned}
\text { Maximize } & -\frac{1}{2} \sum_{i, j=1}^{n}\left(\alpha_{i}-\alpha_{i}^{*}\right)\left(\alpha_{j}-\alpha_{j}^{*}\right)\left\langle\phi\left(\mathbf{x}_{i}\right), \phi\left(\mathbf{x}_{j}\right)\right\rangle \\
& -\varepsilon \sum_{i=1}^{n}\left(\alpha_{i}+\alpha_{i}^{*}\right)+\sum_{i=1}^{n} y_{i}\left(\alpha_{i}-\alpha_{i}^{*}\right)
\end{aligned}
$$

$$
\begin{array}{ll}
\text { Subject to } & \sum_{i=1}^{n}\left(\alpha_{i}-\alpha_{i}^{*}\right)=0 \\
& 0 \leq \alpha_{i}, \alpha_{i}^{*} \leq C,
\end{array}
$$

where $\alpha_{i}, \alpha_{i}^{*}$ are the Lagrange multipliers, which can be obtained from the above optimization problem. The weight vector $\mathbf{w}$ can be solved and written as

$$
\mathbf{w}=\sum_{i=1}^{n}\left(\alpha_{i}-\alpha_{i}^{*}\right) \varphi\left(\mathbf{x}_{i}\right) .
$$

For nonlinear regression problems, SVR transforms data to a high-dimensional feature space by a kernel function. The linear SVR algorithm conducted in feature space represents the nonlinear SVR operation in original space. Inner product in the feature space calculated using a kernel function is expressed as

$$
K\left(\mathbf{x}_{i}, \mathbf{x}_{j}\right)=\left\langle\phi\left(\mathbf{x}_{i}\right), \phi\left(\mathbf{x}_{j}\right)\right\rangle .
$$

Radial-basis kernel function (RBF) is a reasonable choice of kernel functions since it equips with more flexible and fewer parameters. It is applied and listed as follows:

$$
K\left(\mathbf{x}_{i}, \mathbf{x}_{j}\right)=\exp \left(-\gamma\left\|\mathbf{x}_{i}-\mathbf{x}_{j}\right\|^{2}\right) .
$$

Therefore, the nonlinear regression function can be calculated and expressed by

$$
f(x)=\sum_{i=1}^{n}\left(\alpha_{i}-\alpha_{i}^{*}\right) K\left(\mathbf{x}_{i}, \mathbf{x}\right)+b .
$$

In the formulation of SVR model, selection of hyperparameters $(C, \gamma, \varepsilon)$ is crucial to improve the generalization ability and prediction accuracy. Grid search method is applied to optimize the hyperparameters. For each combination of the hyperparameters, SVR is trained using the training data and their performance is evaluated by a cross-validation scheme. Optimal hyperparameters are determined to construct the SVR model for modeling the relationship between modal frequencies and nonuniformly distributed temperatures.

2.5. Prediction Capability Evaluation Index. The prediction capabilities of formulated models (MLR, BPNN, and SVR) are examined and compared using training and test set. Prediction error (PE) is proposed to reflect the difference between target and prediction values. $\mathrm{PE}$ is defined as

$$
\mathrm{PE}=f_{i}-\widehat{f}_{i}
$$

where $f_{i}$ is identified modal frequency and $\widehat{f}_{i}$ is predicted modal frequency.

In order to quantify and rank the performances of formulated models, two statistical indicators including root mean squared error (RMSE) and correlation coefficient $(R)$ are used to quantitatively evaluate the performance of regression models, which are expressed by (16), and $R$ is a numerical value between -1 and 1 , which illustrates the relationship between target and predicted values. A high $R$ value close to 1 indicates a strong positive correlation between target and predicted values, which demonstrates good generalization capability of models. RMSE represents the root mean square of differences between actual and predicted values. It can be also used to evaluate forecasting accuracy of models.

$$
\begin{aligned}
\text { RMSE } & =\sqrt{\frac{\sum_{i=1}^{n}\left(f_{i}-\widehat{f}_{i}\right)^{2}}{n}} \\
R & =\frac{\operatorname{cov}(f, \widehat{f})}{\sigma(f) \sigma(\widehat{f})},
\end{aligned}
$$

where $n$ is the number of sample data, $\operatorname{cov}(f, \widehat{f})$ represents the covariance between target value $f$ and predicted value $\hat{f}$, and $\sigma(f), \sigma(\widehat{f})$ are standard deviations of $f$ and $\widehat{f}$, respectively.

\section{Case Study}

3.1. Monitoring of Concrete Beam. A RC beam with crosssectional dimensions of $4000 \mathrm{~mm} \times 300 \mathrm{~mm} \times 400 \mathrm{~mm}$ is constructed and placed outside the laboratory in Changchun City, China. It is simply supported on concrete piers by two bearings, as shown in Figure 2. The measured span between two bearings is $3700 \mathrm{~mm}$, and $150 \mathrm{~mm}$ overhangs are present 


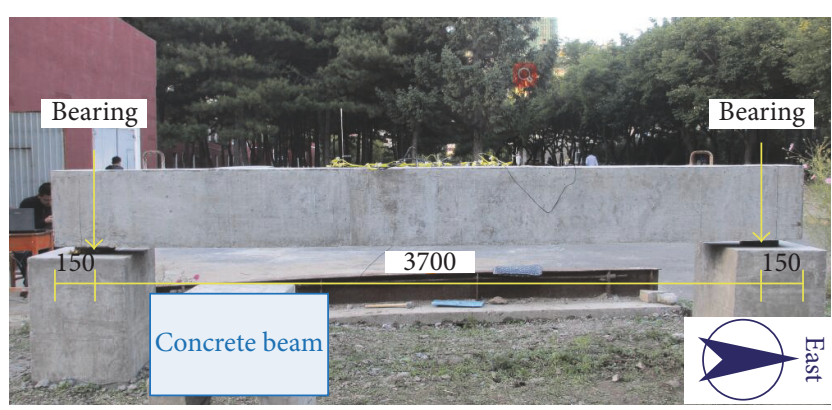

FIGURE 2: RC beam constructed for monitoring (unit: $\mathrm{mm}$ ).

at each end. The RC beam was produced on 8 June 2015 and was installed on 12 September 2015. During this period, the hydration reaction is sufficiently completed, and shrinkage and creep of concrete would produce negligible effect on measurement results. Considering that the nonuniform temperature is primarily distributed in cross-section, a total of 14 type $\mathrm{T}$ thermocouples are embedded in the cross-section at the mid-span of the beam to monitor the temperature field. Figure 3 illustrates the deployment of thermocouples at mid-span. "V" represents thermocouples along vertical direction, while " $\mathrm{H}$ " represents the thermocouples along horizontal direction. In order to fix the thermocouples in accurate position, preformed concrete units containing type $\mathrm{T}$ thermocouples are located at predetermined position before pouring concrete. Type $\mathrm{T}$ thermocouples are made by copper and constantan, and measurement range of them is -250 to $260^{\circ} \mathrm{C}$. A TP700 multichannel data recorder is employed to sample data collected by type $\mathrm{T}$ thermocouples. It features an auto-zero channel, a cold-junction compensator, and automatic voltage-temperature conversions for common thermocouples. Due to the operating temperature ranging from 0 to $50^{\circ} \mathrm{C}$, it is installed in laboratory to ensure the accuracy of measurement. Type $\mathrm{T}$ thermocouples are connected to TP700 data recorder using shielded thermocouple compensation lead [39].

As for the modal testing, two DH131E piezoelectric accelerometers are used to acquire the acceleration response under impact excitation from a rubber hammer at 7/10 span length from left end along vertical direction. The $1^{\#}$ accelerometer is placed at mid-span and $2^{\#}$ accelerometer at $3 / 10$ span length from left end. DH131E accelerometers feature a sensitivity of $1 \mathrm{mV} / \mathrm{g}$, a frequency range of $1-8000 \mathrm{~Hz}$, small size $(\varphi 10 \times$ $16 \mathrm{~mm}$ ), and light weight $(5.5 \mathrm{~g})$. The operating temperature ranging from -40 to $80^{\circ} \mathrm{C}$ makes it ideal for outdoor application. The magnetic bases are fixed on the upper surface of $\mathrm{RC}$ beam using metal/concrete epoxy, and accelerometers are mounted on the magnetic bases. A DH5922 type dynamic signal measurement and analysis system is used to measure and analyze acceleration response. It includes sixteen 24-bit Integrated Electronics PiezoElectric (IEPE) input channels and supports sampling rates of up to $51.2 \mathrm{kHz}$. Antialiasing filters and time-base export for tight synchronization between channels are equipped. The DH5922 system has an operating temperature range of 0 to $40^{\circ} \mathrm{C}$, and it is placed in laboratory. Accelerometers are connected with data acquisition device using L5 coaxial extension cables with lengths of $15 \mathrm{~m}$. DH5922 system samples a 16-second data from the two acceleration channels at a $5120 \mathrm{~Hz}$ sampling rate. Data processing is performed by DHDAS-2013 software platform, which is an important part of DH5922 system. Firstly, the recorded sample data is bandpass-filtered between 10 and $1000 \mathrm{~Hz}$ using a finite impulse response filter. Secondly, Hamming window with $50 \%$ overlap is used to intercept acceleration signal. The number of spectrum lines is set as 6400 , and obtained frequency resolution is $0.156 \mathrm{~Hz}$. Finally, modal frequencies are identified and extracted by frequency spectrum analysis using fast Fourier transform (FFT). For example, samples of data collected from accelerometers installed on the RC beam on March 6, 2016, at 8:00 am are shown in Figure 4. Both the time history of acceleration signal and corresponding amplitude spectrum are illustrated.

Vibration tests were carried out with two hour intervals from 8:00 am to 22:00 pm in everyday monitoring, while temperatures are measured at an interval of 5 minutes. The continuously measured temperatures are used to analyze the temperature variation and its nonuniform distribution. In this study, temperatures corresponding to vibration rests are selected and employed to explore the correlation between temperature and modal frequency and construct regression models between them.

3.2. Temperature Effect on Modal Frequencies. Due to the restrictions of measurement equipment, $\mathrm{RC}$ beams are discontinuously monitored. Measurement data have been collected during the period beginning on 20 September 2015 and ending on 29 August 2016. In this research, measurement data from forty days of monitoring since 19 January 2016 covering winter (minimum temperature) and summer (maximum temperature) are used. During this period, measurements are carried out under weak wind condition (hourly-average wind speed less than $3 \mathrm{~m} / \mathrm{s}$ ). Therefore, wind speed effect on modal frequency can be ignored.

Under the influence of solar radiation, air temperature, and thermal inertia of concrete, temperature distributions in the RC beam are usually nonuniform and nonlinear. In order to specifically exhibit the nonuniform temperature distributions in beam, temperatures measured on 27 June 2016 at 14:00 pm are shown in Figure 5. One can find that the temperature distributions along vertical direction and horizontal direction are nonuniform and nonlinear. This indicated that it is important to consider the nonuniformly distributed temperatures in modeling the relationship between modal frequencies and temperatures.

A total of 320 sets of modal frequencies and temperature data from January to August in 2016 are obtained. The measured data samples are sorted in measurement time order. Figure 6 presents the identified modal frequencies and measured average temperature during monitoring period. As can be seen, significant negative correlations exist between modal frequencies and temperature. The modal frequencies decrease with average temperature increasing. Statistical analysis for the variation of modal frequencies during monitoring period is summarized in Table 1. Average temperature variation ranging from $-22^{\circ} \mathrm{C}$ to $37^{\circ} \mathrm{C}$ accounts for the change 


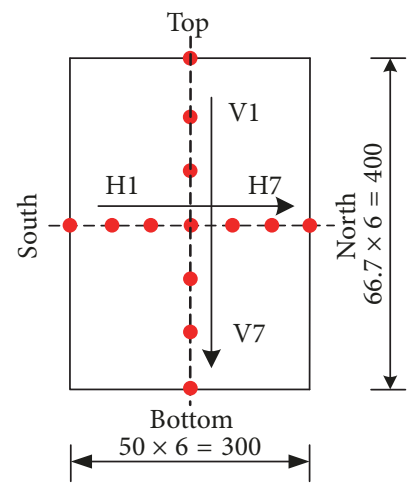

(a)

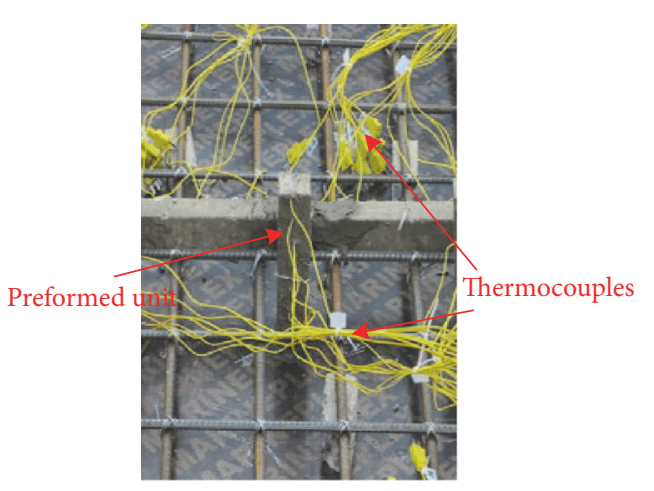

(b)

FIgURE 3: Arrangement and positioning of thermocouples. (a) Arrangement; (b) positioning.

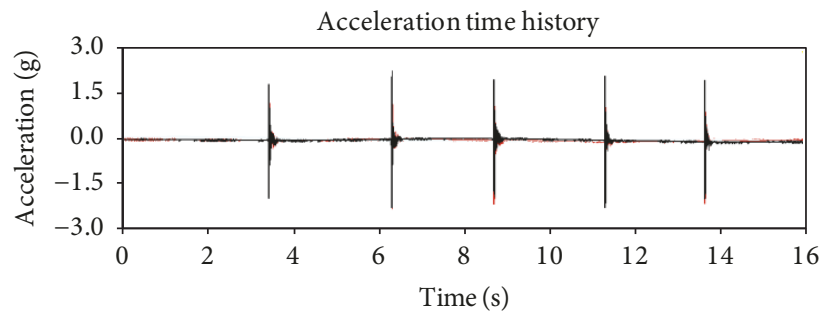

$1^{\#}$ accelerometer

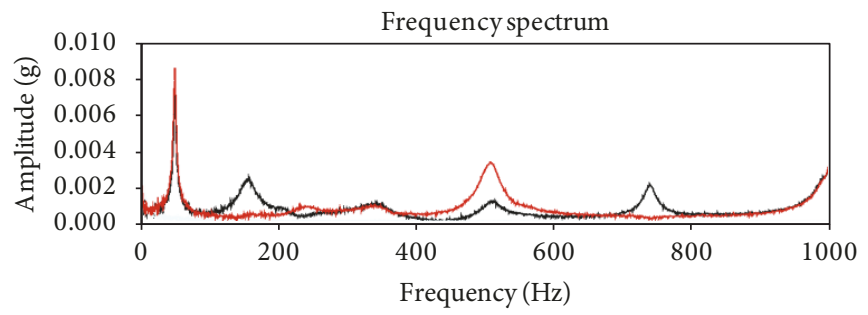

$1^{\#}$ accelerometer
$-2^{\#}$ accelerometer

FIGURE 4: Acceleration time history and corresponding amplitude spectrum.

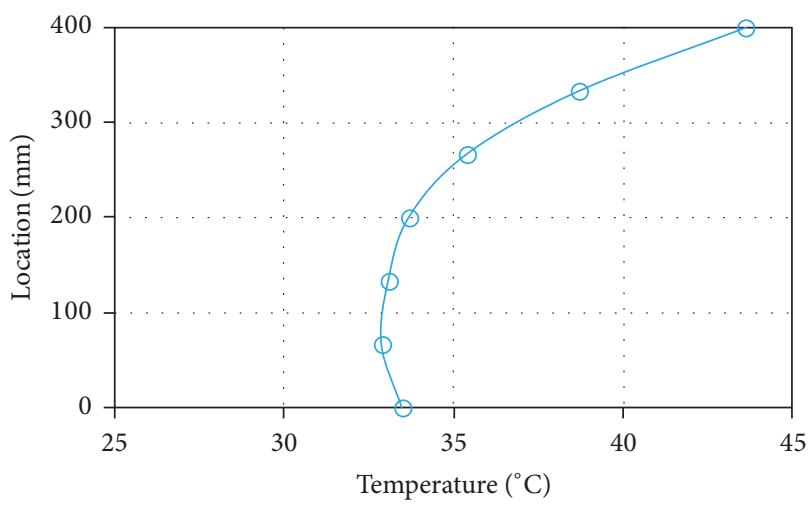

(a)

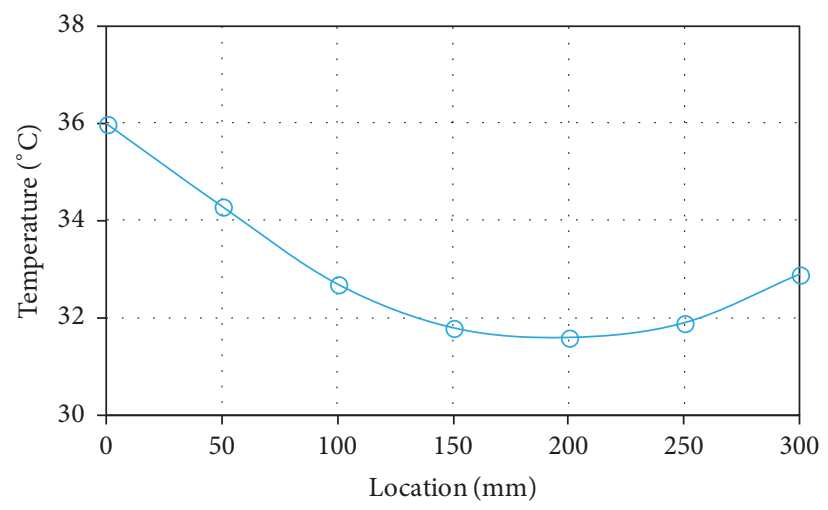

(b)

Figure 5: Temperature distribution on 27 June 2016 at 14:00 pm. (a) Along vertical direction; (b) along horizontal direction. 


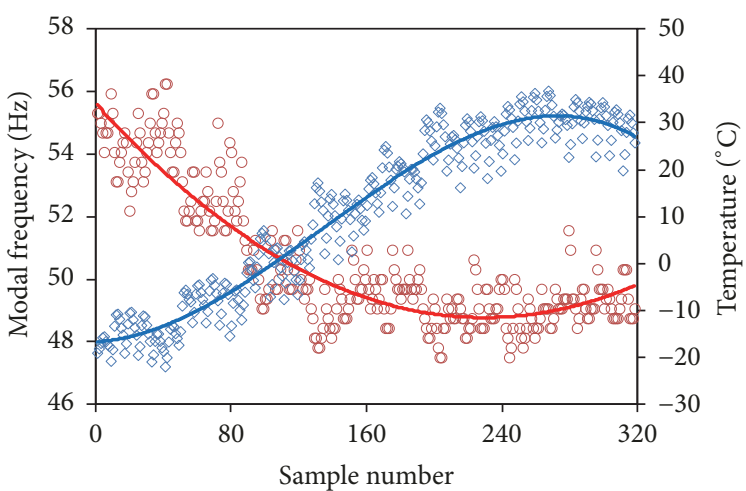

- Modal frequency

$\diamond$ Temperature

(a)

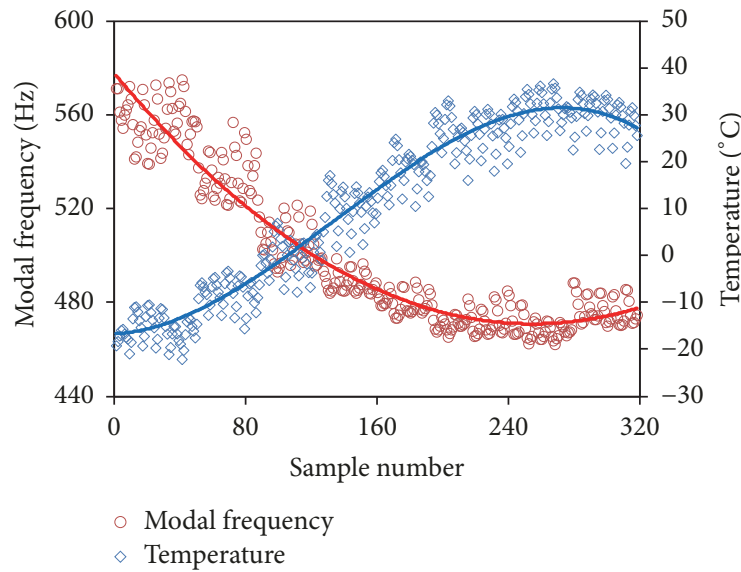

(c)

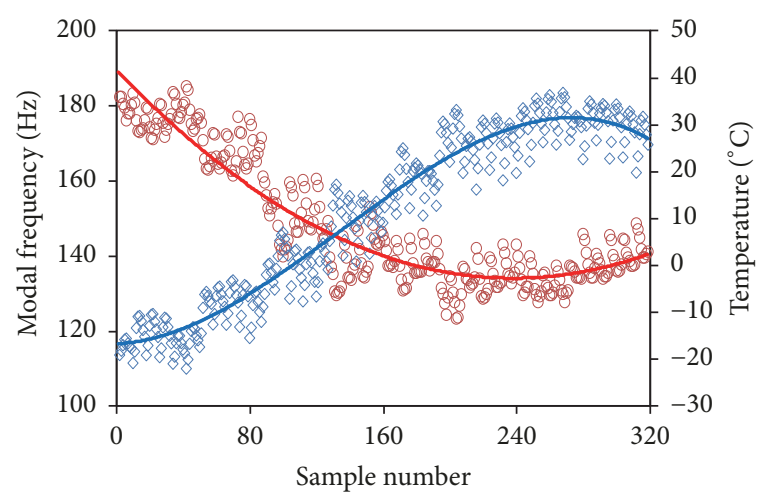

- Modal frequency

$\diamond$ Temperature

(b)

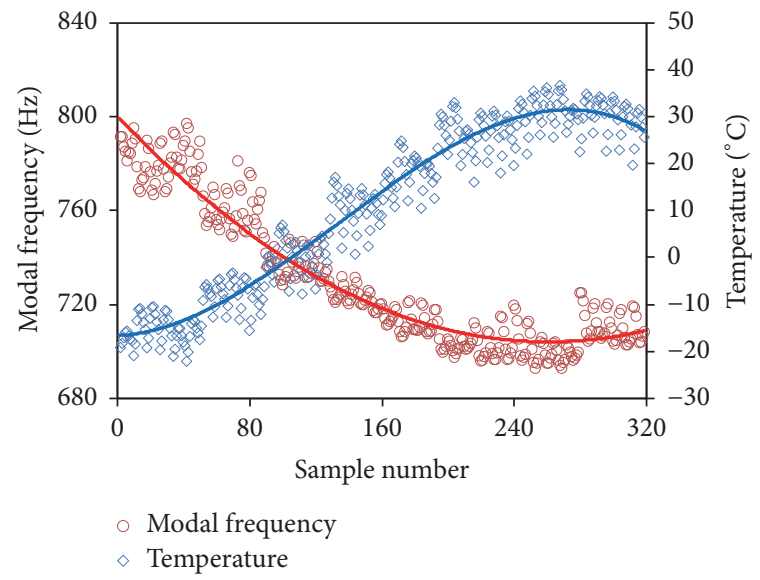

(d)

FIGURE 6: Modal frequencies (red) versus average temperature (blue) (thick lines represent the fitting curve). (a) 1st mode; (b) 2nd mode; (c) 3rd mode; (d) 4th mode.

in modal frequencies of $14.29 \%$ to $41.70 \%$ in the relative sense for the first four modes. And the variation coefficient varies from $4.05 \%$ to $11.48 \%$. For the second modal frequency, it is combined action from vertical vibration and torsional vibration interfered. The higher relative variation (41.70\%) and variation coefficient $(11.48 \%$ ) could be caused by the couple effect of vertical vibration and torsional vibration. As listed in Table 1, significant changes of first four modal frequencies demonstrate the necessity to eliminate temperature effect.

\section{Performance Comparison between Regression Models}

4.1. Formulation of Regression Models. Three regression algorithms presented in previous section are applied to predict modal frequencies of RC beam, and prediction accuracy of models becomes the utmost concern. Table 1 indicates that the ranges of variation of modal frequencies vary with modes. If models are configured to accommodate all the modes, corresponding reproduction and prediction capabilities will be reduced [40]. Therefore, an individual model is developed for each mode separately, which will improve the accuracy of predicting and eliminating temperature effect on modal frequencies. Measurement data from 40 days of monitoring on RC beam are divided into three nonoverlapping and independent data sets: a training set of 50\% (160 sets of data from 20 days of monitoring), a validation set of $20 \%$ (64 sets of data from 8 days of monitoring), and a test set of 30\% (96 sets of data from 12 days of monitoring). Training set is used to construct and train the regression models, validation set to optimize the models, and test set to check their prediction accuracy. The detailed partition is illustrated in Figure 7 taking the first modal frequency, for example. Training set covers a complete temperature range, which is necessary to contain the limitation of prediction. Validation set and test set are uniformly distributed in the range of training set. This partition is helpful to improve the accuracy of prediction models.

In multivariate regression, the highly correlated data could produce unstable regression estimates [3,35]. Internal temperatures of RC beam measured by thermocouples embedded in the cross-section at mid-span are highly correlated. In this research, PCA technique is employed to extract the PCs of temperatures taken from all thermocouples over 
TABLE 1: Statistics of first four modal frequencies.

\begin{tabular}{|c|c|c|c|c|c|c|}
\hline Mode & $\begin{array}{l}\mathrm{Max} \\
(\mathrm{Hz})\end{array}$ & $\begin{array}{l}\text { Min } \\
(\mathrm{Hz})\end{array}$ & $\begin{array}{c}\text { Mean } \\
(\mathrm{Hz})\end{array}$ & $\begin{array}{c}\text { Relative } \\
\text { variation (\%) }\end{array}$ & $\begin{array}{c}\text { Standard } \\
\text { deviation } \\
(\mathrm{Hz})\end{array}$ & $\begin{array}{c}\text { Variation } \\
\text { coefficient } \\
(\%)\end{array}$ \\
\hline 1st & 56.25 & 47.50 & 50.51 & 17.32 & 2.16 & 4.28 \\
\hline 2nd & 185.31 & 123.49 & 148.38 & 41.70 & 17.04 & 11.48 \\
\hline $3 \mathrm{rd}$ & 575.31 & 462.5 & 499.63 & 22.58 & 32.67 & 6.54 \\
\hline 4 th & 797.50 & 693.13 & 730.30 & 14.29 & 29.56 & 4.05 \\
\hline
\end{tabular}

Note. Relative variation $=(\operatorname{Max}-\mathrm{Min}) /$ Mean $* 100 \%$.

TABLE 2: RMSE and $R$ values of reproduced modal frequencies.

\begin{tabular}{llccccccc}
\hline \multirow{2}{*}{ Model } & \multicolumn{2}{c}{ 1st mode } & \multicolumn{2}{c}{ 2nd mode } & \multicolumn{2}{c}{ 3rd mode } & \multicolumn{2}{c}{ 4th mode } \\
& RMSE & $R$ & RMSE & $R$ & RMSE & $R$ & RMSE \\
\hline MLR & 0.8413 & 0.9163 & 5.0748 & 0.9427 & 9.0764 & 0.9547 & 7.7886 \\
BPNN & 0.4072 & 0.9839 & 3.0462 & 0.9849 & 3.8317 & 0.9935 & 3.6636 \\
SVR & 0.3234 & 0.9910 & 2.4426 & 0.9916 & 2.8059 & 0.9965 & 2.6063 & 0.9939 \\
\hline
\end{tabular}

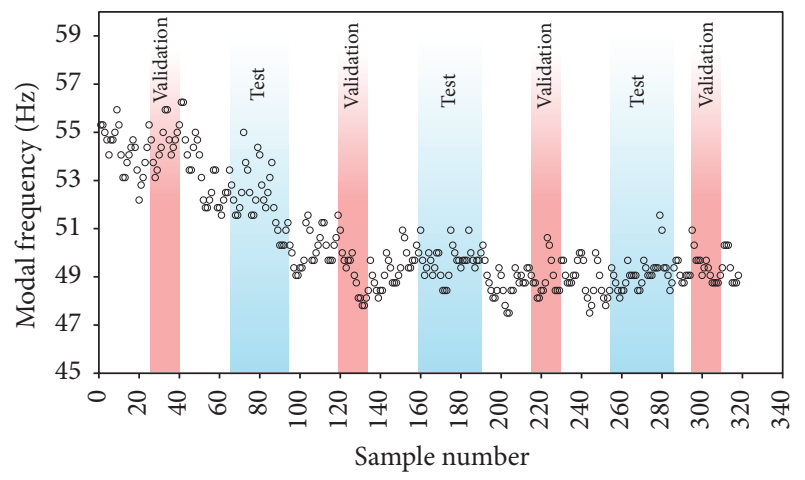

FIgURE 7: Sample selection for training, validation, and test set.

the monitoring period, and the transformed data is then given as input to the regression models. The 320 sets of temperatures measured by 14 thermocouples are analyzed by PCA. Variance contribution rate of the first four PCs accounts for $99.99 \%$ of total variance, and hence the extracted four PCs of temperatures are applied to construct regression models for accurately predicting modal frequencies.

The relationship between modal frequencies and PCs of temperatures are first formulated for each mode by MLR. Training data set is used to build MLR models and calculate regression coefficients using the least square method. For the formulation of BPNN model, an early stopping technique is employed to optimize the BPNN model and avoid overfitting caused by unreasonable performance goal. With intent to determine the optimal number of hidden nodes, BPNN models with different number of hidden nodes are trained by the early stopping technique using training data. The optimal number of hidden nodes is determined so that the validation error reaches the minimum value. Optimally configured BPNN model is selected as the one with 5 hidden nodes for the first vibration mode. Similarly, the optimal numbers of hidden nodes for the other modes are determined as
4, 4, and 5, respectively. SVR modeling is also carried out individually for each mode using the LIBSVM toolbox in MATLAB [41]. A grid search method is used to determine the optimal hyperparameters $(C, \gamma, \varepsilon)$. The bounds on the hyperparameters $C$ and $\gamma$ are set to vary from $2^{-10}$ to $2^{10}$, and $\varepsilon$ is set to vary from 0 to $10^{-3}$. SVR models are built by the training data set using fivefold cross-validation scheme.

4.2. Reproduction Capability. PEs between identified and reproduced modal frequencies are calculated on training set and are evaluated by the use of histograms. Figure 8 presents the histograms of PEs generating from the three regression models. As can been seen, the PEs generated by BPNN and SVR model are concentrated in narrower range, and the observed probability distribution is in good agreement with a normal distribution with zero mean. It indicates that the reproduction capacities of formulated BPNN and SVR models are excellent compared to MLR model. Additionally, the PEs from SVR model are smaller than BPNN model, and the distributions are concentrated in zero more significantly, which demonstrates the outstanding reproduction capability of SVR model.

RMSE and $R$ values between target and reproduced modal frequencies for the first four modes are listed in Table 2. It is observed that the MLR model generates the highest RMSE among the three models, while the SVR model achieves the lowest. In addition, $R$ values of the SVR model are larger than those of MLR and BPNN models. This reveals that SVR model presents a stronger linear relationship between reproduced and identified modal frequencies. Based on above comparisons, SVR model has higher accuracy in reproducing the training data and the reproduction capability rank with a descending order of SVR, BPNN, and MLR.

4.3. Prediction Capability. The prediction capacities of formulated models are verified using testing data set. Histograms of PEs generating from the three regression models 
TABLE 3: RMSE and $R$ values of predicted modal frequencies.

\begin{tabular}{llcccccrc}
\hline \multirow{2}{*}{ Model } & \multicolumn{2}{c}{ 1st mode } & \multicolumn{2}{c}{ 2nd mode } & \multicolumn{2}{c}{ 3rd mode } & \multicolumn{2}{c}{ 4th mode } \\
& RMSE & $R$ & RMSE & $R$ & RMSE & $R$ & RMSE \\
\hline MLR & 0.9140 & 0.9005 & 5.8713 & 0.9416 & 9.9947 & 0.9465 & 8.0774 \\
BPNN & 0.6553 & 0.9417 & 3.5616 & 0.9766 & 5.6797 & 0.9777 & 6.6021 \\
SVR & 0.6223 & 0.9492 & 2.6851 & 0.9846 & 5.0268 & 0.9826 & 5.5101 & 0.9635 \\
\hline
\end{tabular}

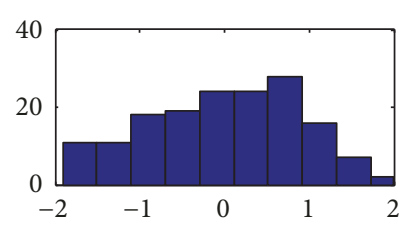

(a1)

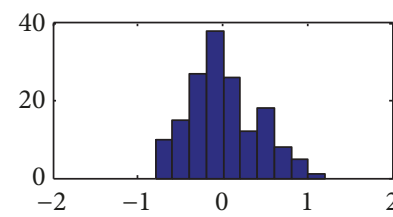

(b1)

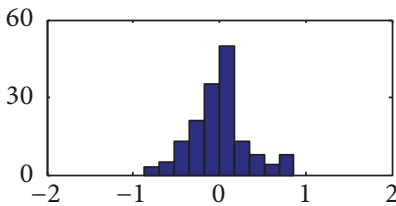

(c1)

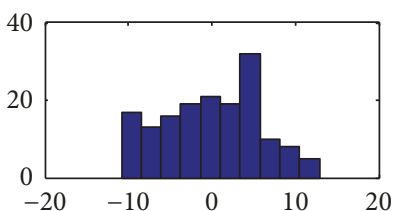

(a2)

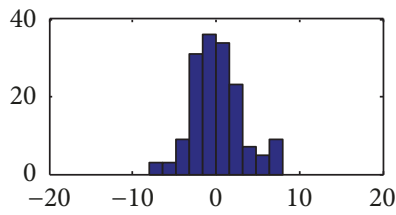

(b2)

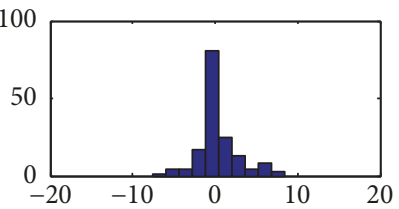

(c2)

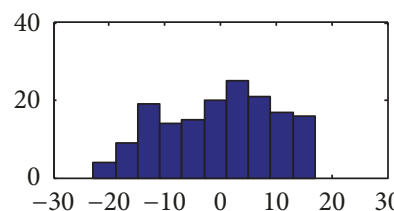

(a3)

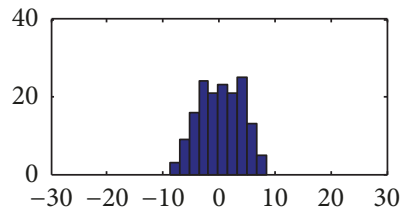

(b3)

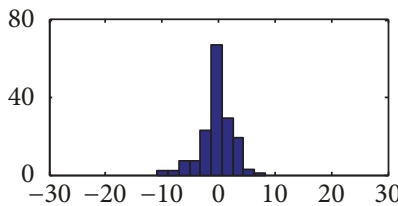

(c3)

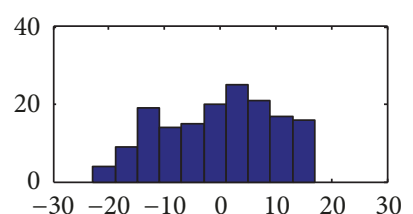

(a4)

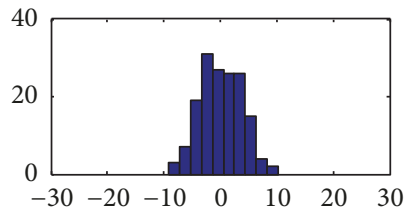

(b4)

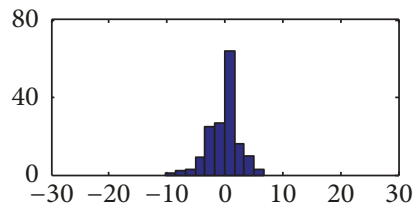

(c4)

FIGURE 8: Histograms of PEs generated by three regression models on training set (horizontal axis represents PEs, and vertical axis represents sample number). (a1) MLR for 1st mode; (a2) MLR for 2nd mode; (a3) MLR for 3rd mode; (a4) MLR for 4th mode; (b1) BPNN for 1st mode; (b2) BPNN for 2nd mode; (b3) BPNN for 3rd mode; (b4) BPNN for 4th mode; (c1) SVR for 1st mode; (c2) SVR for 2nd mode; (c3) SVR for 3rd mode; (c4) SVR for 4th mode.

are illustrated in Figure 9. It presents that the PEs generated by BPNN and SVR model are concentrated in narrower range. And the observed probability distribution is in good agreement with a normal distribution with zero mean. Similar to reproduction capability, BPNN and SVR models possess better prediction accuracy than MLR model because of less prediction error.

RMSE and $R$ values of three regression models for all the modes are listed in Table 3. It can be seen that RMSE values of MLR, BPNN, and SVR models rank with a descending order of MLR, BPNN, and SVR. The SVR model with minimal RMSE value performs higher prediction accuracy than the BPNN and MLR models. Moreover, $R$ values of SVR model are larger than those of other models, which imply that the performance of SVM models is excellent in predicting the modal frequencies under the nonuniformly distributed temperatures.

\section{Eliminating Temperature Effect on Modal Frequency}

The main purpose of constructing accurate regression model is to eliminate the temperature effect on the modal frequencies and to normalize all the modal frequencies to a set of reference temperature. Comparing to the MLR and BPNN regression models, the SVR model exists with better capability for predicting modal frequencies of RC beam in this research. The established SVR model above is used to eliminate the temperature effect on modal frequencies. Firstly, the modal frequencies at the reference temperature of $20^{\circ} \mathrm{C}$ are identified. Then the normalized modal frequencies after removing environmental effect can be obtained by

$$
f=f_{\text {reference }}+\left(f_{\text {identified }}-f_{\text {predicted }}\right)
$$

where $f_{\text {reference }}$ is the modal frequencies at reference temperature, $f_{\text {predicted }}$ is the modal frequencies predicted by SVR regression model, and $f_{\text {identified }}$ represents the modal frequencies identified at different temperature.

Figure 10 illustrates the PEs of first four modal frequencies produced by SVR regression model. It can be clearly observed that the variation of PEs is around zero (the small difference is due to the measurement error, prediction error, and other noise). This indicates that the seasonal variation of modal frequencies is successfully eliminated. Histograms of modal frequencies before and after correction by SVR regression model are plotted to demonstrate the reduction of the variation of modal frequencies. Figure 11 presents the histograms 
TABLE 4: Variability of first four modal frequencies before and after eliminating temperature effect.

\begin{tabular}{|c|c|c|c|c|c|c|}
\hline \multirow{2}{*}{ Mode } & \multicolumn{3}{|c|}{ Standard deviation $(\mathrm{Hz})$} & \multicolumn{3}{|c|}{ Variation coefficient (\%) } \\
\hline & Before & After & Ratio & Before & After & Ratio \\
\hline 1 st & 2.16 & 0.46 & 21.30 & 4.28 & 0.92 & 21.50 \\
\hline 2nd & 17.04 & 2.82 & 16.55 & 11.48 & 2.05 & 17.86 \\
\hline $3 \mathrm{rd}$ & 32.67 & 3.91 & 11.97 & 6.54 & 0.82 & 12.54 \\
\hline 4 th & 29.56 & 4.39 & 14.85 & 4.05 & 0.62 & 15.31 \\
\hline
\end{tabular}

Note. Ratio $=$ after/before $* 100 \%$.

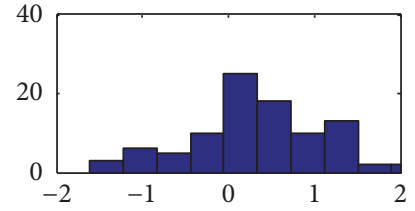

(a1)

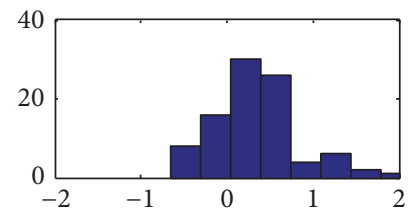

(b1)

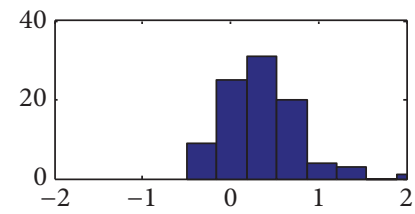

$(\mathrm{c} 1)$

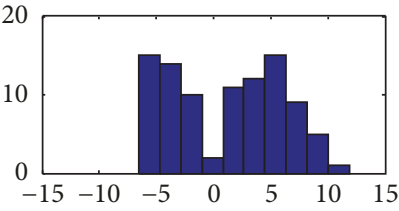

(a2)

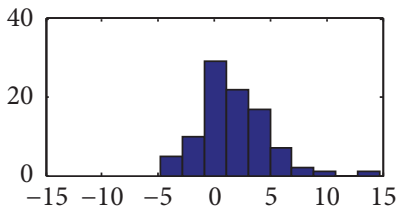

(b2)

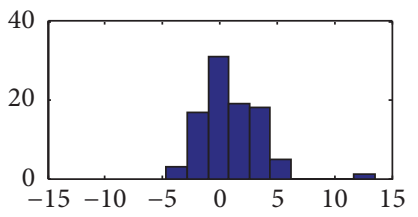

(c2)

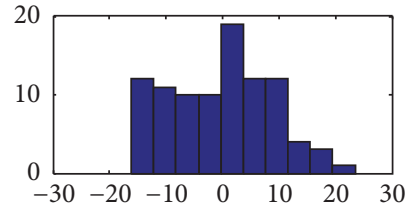

(a3)

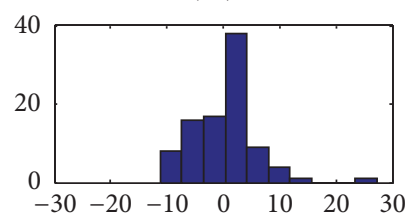

(b3)

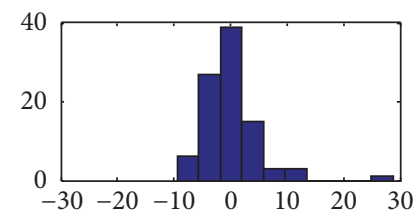

(c3)

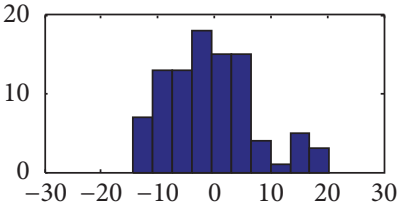

(a4)

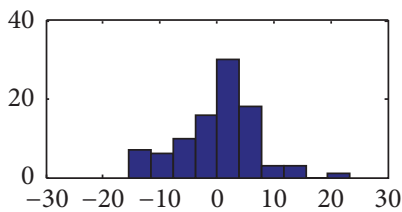

(b4)

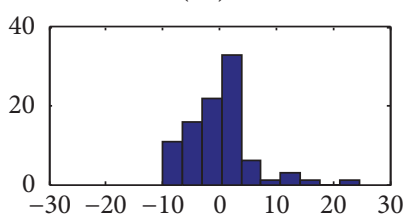

(c4)

Figure 9: Histograms of PEs generated by three regression models on test set (horizontal axis represents PEs, and vertical axis represents sample number). (a1) MLR for 1st mode; (a2) MLR for 2nd mode; (a3) MLR for 3rd mode; (a4) MLR for 4th mode; (b1) BPNN for 1st mode; (b2) BPNN for 2nd mode; (b3) BPNN for 3rd mode; (b4) BPNN for 4th mode; (c1) SVR for 1st mode; (c2) SVR for 2nd mode; (c3) SVR for 3rd mode; (c4) SVR for 4th mode.

of first four modal frequencies during the period of 40 days of monitoring. It is clear that the modal frequencies are concentrated in narrower range with normal distributions after the application of SVR regression model. Standard deviation and variation coefficient of first four modal frequencies before and after eliminating temperature effect are listed in Table 4. As can be seen, standard deviations of modal frequencies after eliminating temperature effect are only $25 \%$ of standard deviations before eliminating temperature effect. Maximum variation coefficient is reduced from $11.48 \%$ to $2.05 \%$ after eliminating temperature effect. This verifies the effectiveness of SVR regression model in eliminating temperature effect on modal frequencies.

\section{Conclusions}

In this paper, three regression models are constructed to predict the modal frequencies of a concrete beam caused by temperature change in seasonal cold region. The prediction capabilities of formulated MLR, BPNN, and SVR models are evaluated and compared. The following conclusions can be obtained.
(1) During the monitoring period, average temperature variation in $\mathrm{RC}$ beam ranging from $-22^{\circ} \mathrm{C}$ to $37^{\circ} \mathrm{C}$ accounts for the changes in first four modal frequencies of $14.29 \%$ to $41.70 \%$ in relative sense. And the variation coefficient ranges from $4.05 \%$ to $11.48 \%$. It demonstrates the necessity to eliminate temperature effect on modal frequency.

(2) A series of statistical indexes including PE, RMSE, and $R$ are introduced to evaluate the reproduction and prediction capability of the formulated models. Histograms statistics of PEs demonstrates that the reproduction and prediction capability of SVR model are superior to MLR and BPNN models. Comparison analysis on RMSE and R indicators also prove that SVR model exhibits excellent reproduction and prediction capabilities and evaluates the modal frequency with high accuracy.

(3) Eliminating temperature effect on modal frequencies is achieved by use of the established SVR model. After eliminating temperature effect, seasonal variation of modal frequencies disappeared, and modal frequencies are concentrated in narrower range with normal distributions. Comparison between variabilities of modal frequencies before and after eliminating temperature effect demonstrates 


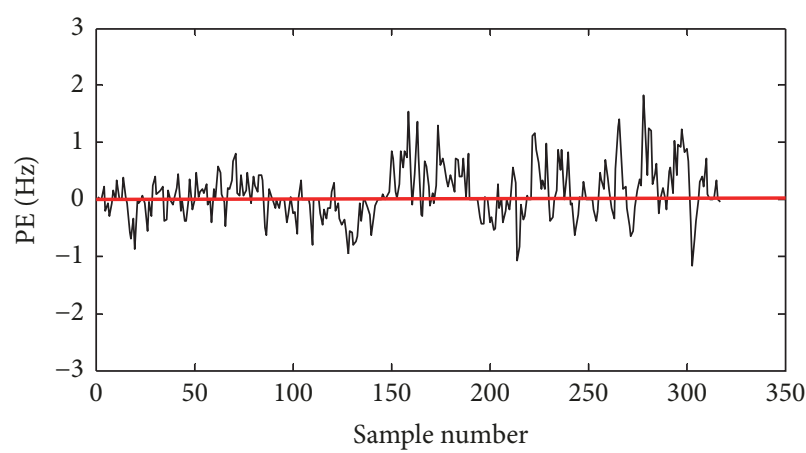

(a)

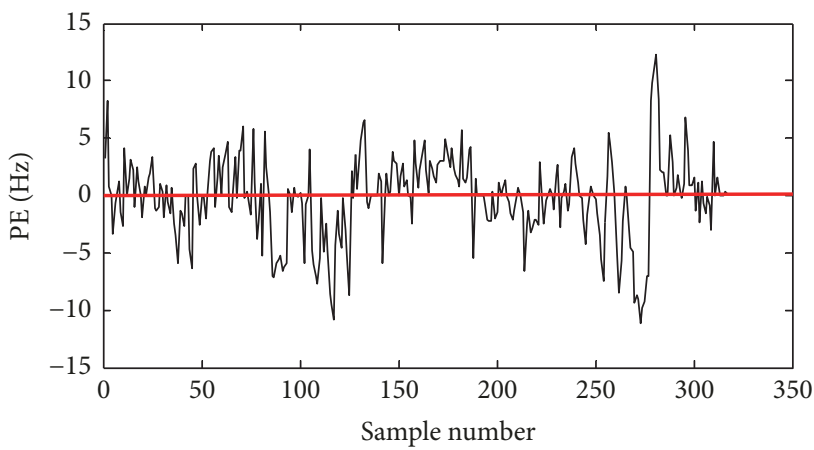

(c)

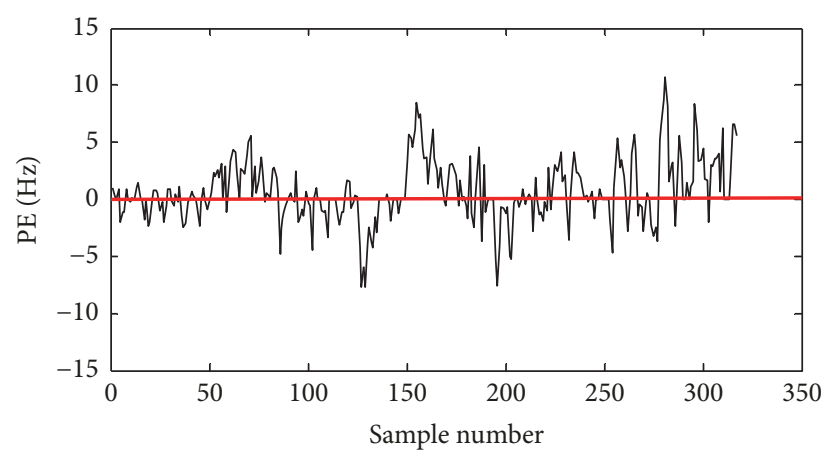

(b)

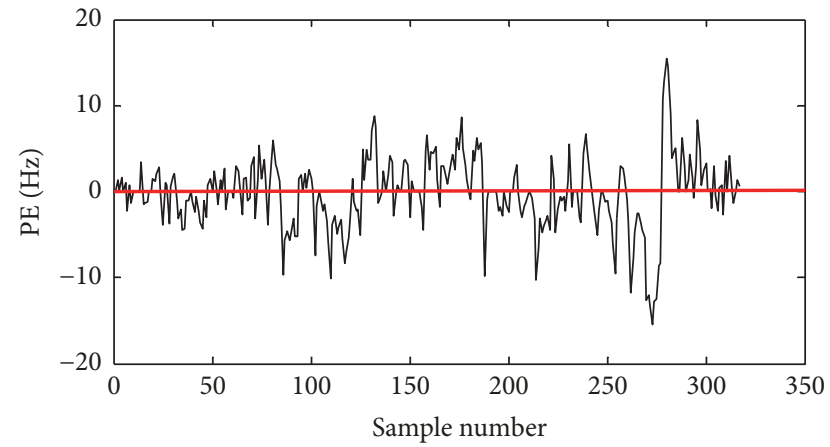

(d)

FIGURE 10: PEs of first four modal frequencies by use of SVR model. (a) 1st mode; (b) 2nd mode; (c) 3rd mode; (d) 4th mode.

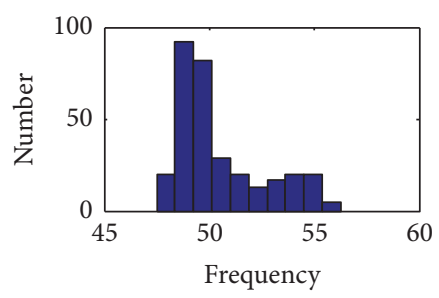

(a1)

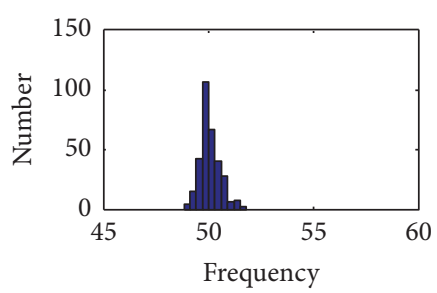

(b1)

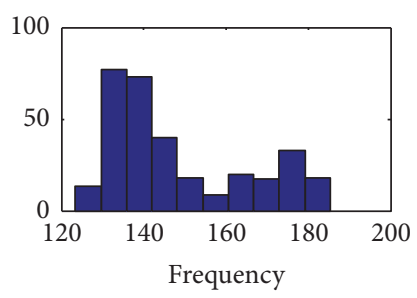

(a2)

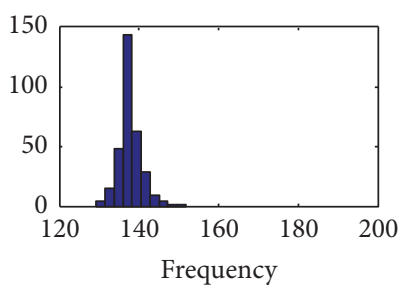

(b2)

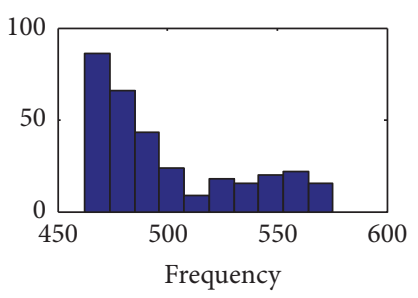

(a3)

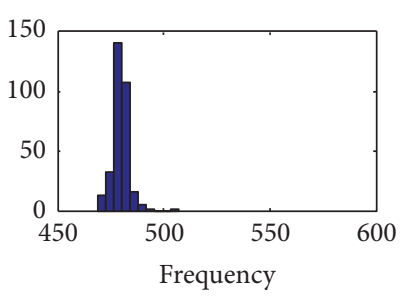

(b3)

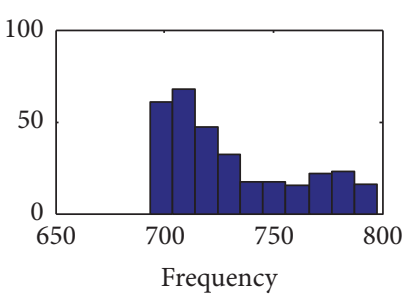

(a4)

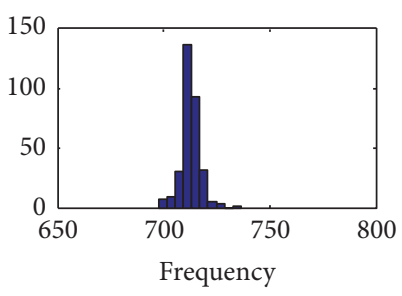

(b4)

FIGURE 11: Histograms of the modal frequencies before and after the elimination of temperature effects. (al) 1st modal frequency before eliminating temperature effect; (a2) 2nd modal frequency before eliminating temperature effect; (a3) 3rd mode modal frequency before eliminating temperature effect; (a4) 4th modal frequency before eliminating temperature effect; (b1) 1st modal frequency after eliminating temperature effect; (b2) 2nd modal frequency after eliminating temperature effect; (b3) 3rd modal frequency after eliminating temperature effect; (b4) 4th modal frequency after eliminating temperature effect.

the effectiveness of SVR model in eliminating temperature effect.

\section{Conflicts of Interest}

The authors declare no conflicts of interest.

\section{Acknowledgments}

The authors express their appreciation for financial supports of National Natural Science Foundation of China under Grants nos. 51408258 and 51378236; China Postdoctoral Science Foundation funded projects (nos. 2014M560237 and 
2015T80305); Fundamental Research Funds for the Central Universities (JCKYQKJC06); Science and Technology Development Program of Jilin Province.

\section{References}

[1] Z. W. Chen, Y. L. Xu, and X. M. Wang, "SHMS-based fatigue reliability analysis of multiloading suspension bridges," Journal of Structural Engineering, vol. 138, no. 3, pp. 299-307, 2011.

[2] K. Y. Wong, "Instrumentation and health monitoring of cablesupported bridges," Structural Control and Health Monitoring, vol. 11, no. 2, pp. 91-124, 2004.

[3] X. G. Hua, Y. Q. Ni, J. M. Ko, and K. Y. Wong, "Modeling of temperature-frequency correlation using combined principal component analysis and support vector regression technique," Journal of Computing in Civil Engineering, vol. 21, no. 2, pp. 122135, 2007.

[4] J. M. Ko and Y. Q. Ni, “Technology developments in structural health monitoring of large-scale bridges," Engineering Structures, vol. 27, no. 12, pp. 1715-1725, 2005.

[5] S. W. Doebling, C. R. Farrar, and M. B. Prime, "A summary review of vibration-based damage identification methods," The Shock and Vibration Digest, vol. 30, no. 2, pp. 91-105, 1998.

[6] G.-D. Zhou and T.-H. Yi, "A summary review of correlations between temperatures and vibration properties of long-span bridges," Mathematical Problems in Engineering, vol. 2014, Article ID 638209, 19 pages, 2014.

[7] D. J. Pines and A. E. Aktan, "Status of structural health monitoring of long-span bridges in the United States," Progress in Structural Engineering and Materials, vol. 4, no. 4, pp. 372380, 2002.

[8] H. Sohn, "Effects of environmental and operational variability on structural health monitoring," Philosophical Transactions of the Royal Society A: Mathematical, Physical \& Engineering Sciences, vol. 365, no. 1851, pp. 539-560, 2007.

[9] D. F. Giraldo, S. J. Dyke, and J. M. Caicedo, "Damage detection accommodating varying environmental conditions," Structural Health and Monitoring, vol. 5, no. 2, pp. 155-172, 2006.

[10] H. Li, S. Li, and J. Ou, "Modal identification of bridges under varying environmental conditions: temperature and wind effects," Structural Control and Health Monitoring, vol. 17, no. 5, pp. 495-512, 2010.

[11] C. Yang and S. O. Oyadiji, "Damage detection using modal frequency curve and squared residual wavelet coefficients-based damage indicator," Mechanical Systems and Signal Processing, vol. 83, pp. 385-405, 2017.

[12] Y. Xia, B. Chen, S. Weng, Y.-Q. Ni, and Y.-L. Xu, "Temperature effect on vibration properties of civil structures: a literature review and case studies," Journal of Civil Structural Health Monitoring, vol. 2, no. 1, pp. 29-46, 2012.

[13] P. Cornwell, C. R. Farrar, S. W. Doebling, and H. Sohn, "Environmental variability of modal properties," Experimental Techniques, vol. 23, no. 6, pp. 45-48, 1999.

[14] H. Sohn, M. Dzwonczyk, E. G. Straser, A. S. Kiremidjian, K. Law, and T. Meng, "An experimental study of temperature effect on modal parameters of the Alamosa Canyon Bridge," Earthquake Engineering \& Structural Dynamics, vol. 28, no. 78, pp. 879-897, 1999.

[15] B. Peeters and G. De Roeck, "One-year monitoring of the Z24bridge: environmental effects versus damage events," Earthquake Engineering \& Structural Dynamics, vol. 30, no. 2, pp. 149171, 2001.
[16] J. Maeck, B. Peeters, and G. De Roeck, "Damage identification on the Z24-bridge using vibration monitoring analysis," in Proceedings of the COST F3 Conference on System Identification and Structural Health Monitoring, pp. 233-242, 2000.

[17] S. L. Desjardins, N. A. Londoño, D. T. Lau, and H. Khoo, "Realtime data processing, analysis and visualization for structural monitoring of the confederation bridge," Advances in Structural Engineering, vol. 9, no. 1, pp. 141-157, 2006.

[18] V. Askegaard and P. Mossing, "Long term observation of RCbridge using changes in natural frequency," Nordic Concrete Research, vol. 7, pp. 20-27, 1988.

[19] K.-V. Yuen and S.-C. Kuok, "Ambient interference in long-term monitoring of buildings," Engineering Structures, vol. 32, no. 8, pp. 2379-2386, 2010.

[20] W. H. Chen, Z. R. Lu, W. Lin et al., "Theoretical and experimental modal analysis of the Guangzhou New TV Tower," Engineering Structures, vol. 33, no. 12, pp. 3628-3646, 2011.

[21] A. Saisi, C. Gentile, and M. Guidobaldi, "Post-earthquake continuous dynamic monitoring of the Gabbia Tower in Mantua, Italy," Construction and Building Materials, vol. 81, pp. 101-112, 2015.

[22] C. Gentile, M. Guidobaldi, and A. Saisi, "One-year dynamic monitoring of a historic tower: damage detection under changing environment," Meccanica, vol. 51, no. 11, pp. 2873-2889, 2016.

[23] F. Ubertini, G. Comanducci, N. Cavalagli, A. Laura Pisello, A. Luigi Materazzi, and F. Cotana, "Environmental effects on natural frequencies of the San Pietro bell tower in Perugia, Italy, and their removal for structural performance assessment," Mechanical Systems and Signal Processing, vol. 82, pp. 307-322, 2017.

[24] Y. Xia, H. Hao, G. Zanardo, and A. Deeks, "Long term vibration monitoring of an RC slab: temperature and humidity effect," Engineering Structures, vol. 28, no. 3, pp. 441-452, 2006.

[25] J. T. Kim, C. B. Yun, and J. H. Yi, "Temperature effects on modal properties and damage detection in plate-girder bridges," Advanced Smart Materials and Structures Technology, pp. 504511, 2004.

[26] H. F. Zhou, Y. Q. Ni, and J. M. Ko, "Eliminating temperature effect in structural damage alarming using auto-associative neural networks," Structural Health and Monitoring, vol. 12, pp. 539-548, 2007.

[27] J. Kim, J. Park, and B. Lee, "Vibration-based damage monitoring in model plate-girder bridges under uncertain temperature conditions," Engineering Structures, vol. 29, no. 7, pp. 1354-1365, 2007.

[28] P. Moser and B. Moaveni, "Environmental effects on the identified natural frequencies of the Dowling Hall Footbridge," Mechanical Systems and Signal Processing, vol. 25, no. 7, pp. 2336-2357, 2011.

[29] Y. L. Ding and A. Q. Li, “Temperature-induced variations of measured modal frequencies of steel box girder for a long-span suspension bridge," International Journal of Steel Structures, vol. 11, no. 2, pp. 145-155, 2011.

[30] Y. Q. Ni, X. G. Hua, K. Q. Fan, and J. M. Ko, "Correlating modal properties with temperature using long-term monitoring data and support vector machine technique," Engineering Structures, vol. 27, no. 12, pp. 1762-1773, 2005.

[31] Y. Q. Ni, H. F. Zhou, and J. M. Ko, "Generalization capability of neural network models for temperature- frequency correlation using monitoring data," Journal of Structural Engineering, vol. 135, no. 10, pp. 1290-1300, 2009. 
[32] H. Nandan and M. P. Singh, "Effects of thermal environment on structural frequencies: Part I - A simulation study," Engineering Structures, vol. 81, pp. 480-490, 2014.

[33] Y. Xia, Y.-L. Xu, Z.-L. Wei, H.-P. Zhu, and X.-Q. Zhou, "Variation of structural vibration characteristics versus non-uniform temperature distribution," Engineering Structures, vol. 33, no. 1, pp. 146-153, 2011.

[34] F. Magalhães, A. Cunha, and E. Caetano, "Vibration based structural health monitoring of an arch bridge: from automated OMA to damage detection," Mechanical Systems and Signal Processing, vol. 28, pp. 212-228, 2012.

[35] R. Kromanis and P. Kripakaran, "Predicting thermal response of bridges using regression models derived from measurement histories," Computers \& Structures, vol. 136, pp. 64-77, 2014.

[36] H. Liu, X. Wang, Y. Gong, and Y. Jiao, "Damage identification of urban overpass based on hybrid neurogenetic algorithm using static and dynamic properties," Mathematical Problems in Engineering, vol. 2015, Article ID 404675, 10 pages, 2015.

[37] M. H. Beale, M. T. Hagan, and H. B. Demuth, Neural network toolbox ${ }^{T M}$ user's guide-R2012a, The MathWorks, Inc., 3 Apple Hill Drive Natick, Massachusetts, Mass, USA, 2012.

[38] C. J. C. Burges, "A tutorial on support vector machines for pattern recognition," Data Mining and Knowledge Discovery, vol. 2, no. 2, pp. 121-167, 1998.

[39] H. B. Liu, X. Q. Wang, and Y. B. Jiao, "Effect of temperature variation on modal frequency of reinforced concrete slab and beam in cold regions," Shock and Vibration, vol. 2016, Article ID 4792786, 17 pages, 2016.

[40] H. F. Zhou, Y. Q. Ni, and J. M. Ko, "Constructing input to neural networks for modeling temperature-caused modal variability: mean temperatures, effective temperatures, and principal components of temperatures," Engineering Structures, vol. 32, no. 6, pp. 1747-1759, 2010.

[41] C. Chang and C. Lin, "LIBSVM: a Library for support vector machines," ACM Transactions on Intelligent Systems and Technology, vol. 2, no. 3, article 27, 2011. 


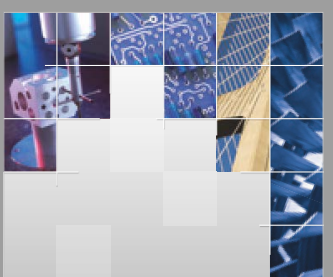

\section{Enfincering}
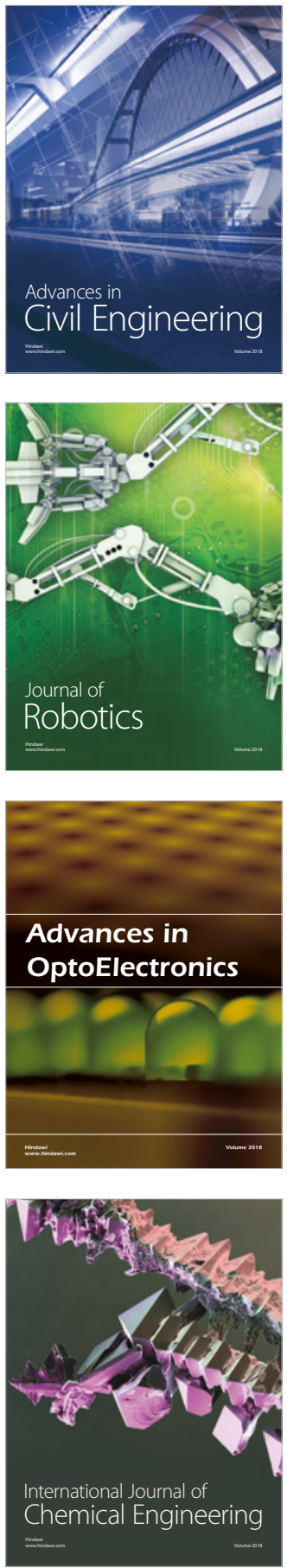

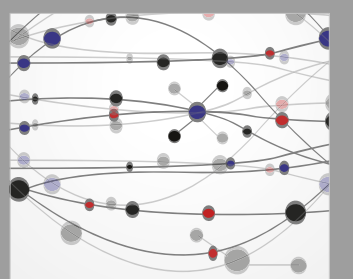

\section{Rotating \\ Machinery}

The Scientific World Journal

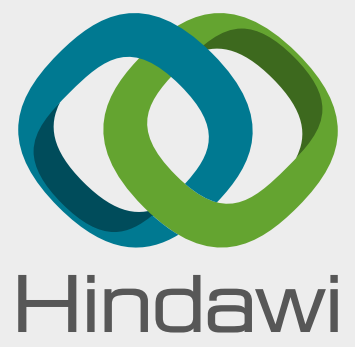

Submit your manuscripts at

www.hindawi.com
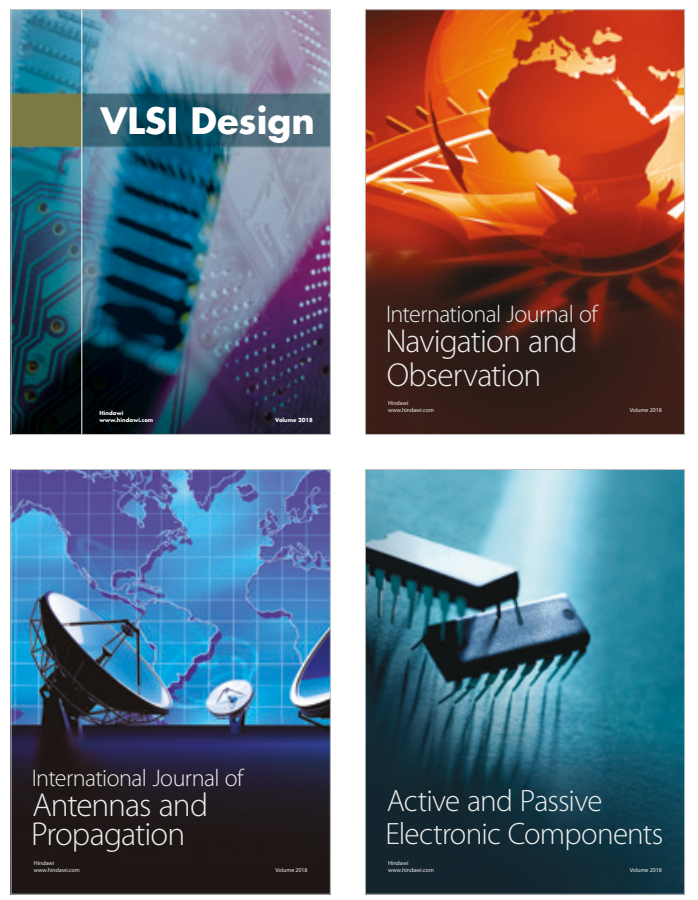
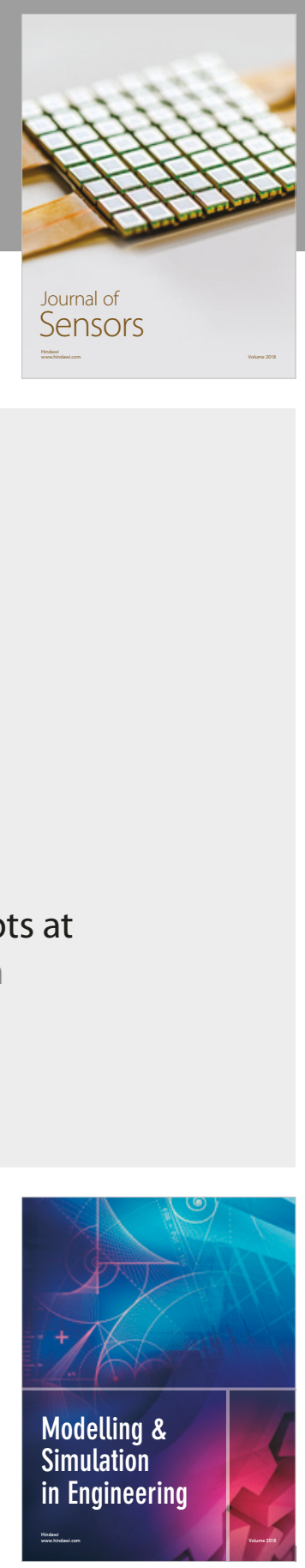

\section{Advances \\ Multimedia}
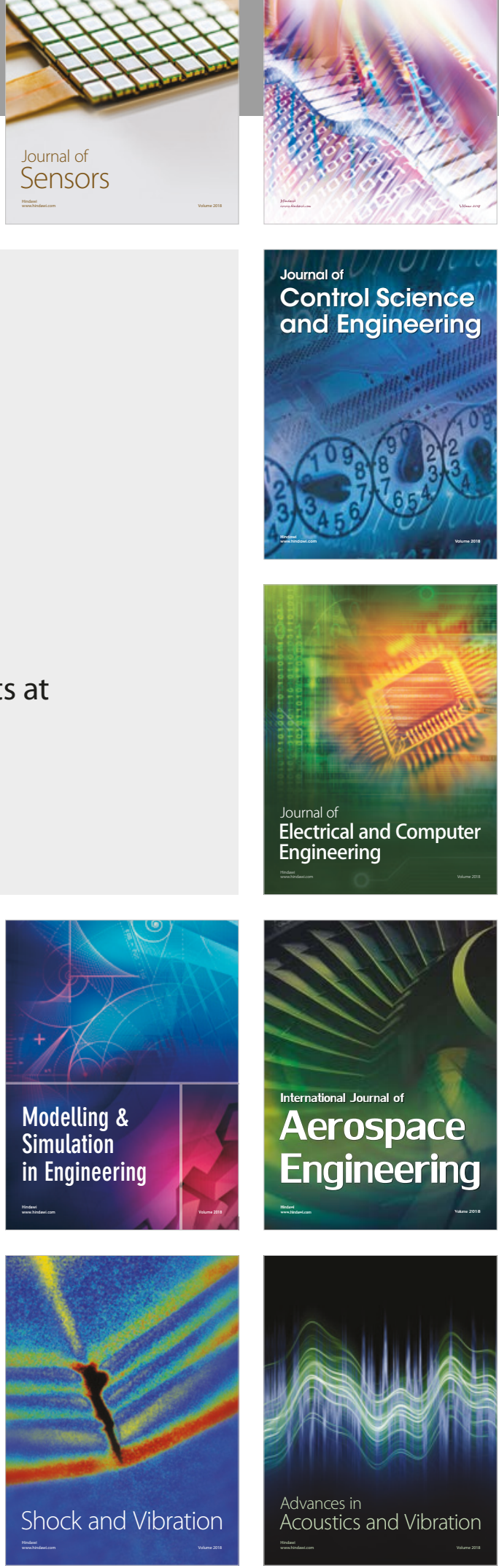